\title{
The Conserved Currents for the Maxwellian Field
}

\author{
Richard Arens \\ Department of Mathematics, University of California, Los Angeles, CA 90024, USA
}

\begin{abstract}
We classify the conserved currents for the Maxwellian field. There are four families: (1) the classical currents derived using Noether's theorem from conformal invariance (2) certain Noetherian currents based on translations in field space, $(3,4)$ two more kinds not equivalent to any Noetherian form.
\end{abstract}

\section{Introduction}

E. Noether first discovered that symmetries for a field theory gave rise to conserved currents (see [2]). The two concepts are not coextensive. We show below that certain well-known conserved currents (the rows of the symmetrized energy-momentum tensor) are not the result, so to speak, of any symmetries of the Maxwellian (electromagnetic) field.

This paper consists of two parts. (1) We enumerate the conserved currents: they fall into four classes. Two of them are the Noetherian forms already known, being those due to the conformal invariance, and the translational invariance in fieldspace (i.e. the transformation of the general field by adding a specific field). The third kind includes the parts added to the current of the first kind when one symmetrizes the energy-momentum tensor. The fourth kind cannot be described in a few words. (2) We prove that the third and fourth kind can never be equivalent to any Noetherian form except in degenerate cases.

As a corollary we obtain a description of all symmetries of the Maxwell system: They are just those already mentioned.

\section{Main Theorem on Dynamic Currents}

We take $\mathbb{R}^{4}$ as our model for space-time $M$ and use $t^{1}, t^{2}, t^{3}, t^{4}$ for the Cartesian coordinates there. We use $u_{1}, u_{2}, u_{3}, u_{4}$ as the Cartesian Coordinates in the space $Q\left(=\mathbb{R}^{4}\right)$, where the field (the 4-vector potential) has its values.

A first order jet from $M$ to $Q$ is a linear map $j$ from some tangent vector space $T^{1}(M, a)$ of $M$ to some $T^{1}(Q, b)$ of $Q$. A function $U$ from $M$ to $Q$ defines a jet $j$ at each $a$ in $M$ with $b=U(a)$. It has coordinates $t^{i}(j)=t^{i}(a), u_{i}(j)=u_{i}(U(a))$. It also has 16 
further coordinates

$$
p_{i j}(j)=\left.\frac{\partial u_{i}(U)}{\partial t^{j}}\right|_{a}
$$

Thus we obtain a 24-dimensional space with coordinates $t^{i}, u_{i}, p_{i j}$ : the first order jet bundle $J^{1}(M, Q)$. See [6].

Let $U_{1}, \ldots, U_{4}$ be a solution of Maxwell's equations:

$$
\left(\frac{\partial^{2} U_{i}}{\partial t^{j} \partial t^{k}}-\frac{\partial^{2} U_{j}}{\partial t^{i} \partial t^{k}}\right) g^{j k}=0
$$

This defines a 4-dimensional submanifold $s$ of $J^{1}(M, Q)$ with the equations

$$
u_{i}=U_{i}, p_{i j}=\frac{\partial U_{i}}{\partial t^{j}}
$$

These submanifolds shall be called motions.

A differential form $\varepsilon$ of degree 3 is called dynamic if its restriction to each motion $s$ is exact. If $\varepsilon$ is of the type

$$
\begin{aligned}
\varepsilon= & J^{1} d t^{2} \wedge d t^{3} \wedge d t^{4}-J^{2} d t^{1} \wedge d t^{3} \wedge d t^{4} \wedge^{\prime}+J^{3} d t^{1} \wedge d t^{2} \wedge d t^{4} \\
& -J^{4} d t^{1} \wedge d t^{2} \wedge d t^{3}
\end{aligned}
$$

then its being dynamic is equivalent to $J^{1}, J^{2}, J^{3}, J^{4}$ being a conserved current. We will then say that $\varepsilon$ is a dynamic current. The results of our study are as follows (observing the usual conventions on summing and on raising and lowering of indices):

2.3 (Theorem). Let (2.2) be a dynamic current for the Maxwell field. Then $J^{i}=\Phi^{i}+\Psi^{i}+\Gamma^{i}+W^{i}+N^{i}$, where

$$
\begin{aligned}
\Phi^{i} & =\left(\frac{\partial A^{j}}{\partial t^{k}} u_{j}+p_{k j} A^{j}\right)\left(p^{i k}-p^{k i}\right)+\frac{1}{2} A^{i} p_{j k}\left(p^{j k}-p^{k j}\right), \\
\Psi^{i} & =\left(f^{j, i}-f^{i, j}\right) u_{j}+f_{j}\left(p^{i j}-p^{j i}\right), \\
\Gamma^{i} & =g^{i} \\
W^{i} & =\left(\frac{\partial S}{\partial t^{j}}+\frac{\partial S}{\partial u_{k}} p_{k j}\right)\left(p^{j i}-p^{i j}\right), \\
N^{i} & =\left(\frac{\partial}{\partial t^{n}}+p_{m n} \frac{\partial}{\partial u_{m}}\right)\left(\theta^{n i}+{ }^{a} \eta^{n i j} p_{a j}+{ }^{a b} \zeta^{n i j k} p_{a j} p_{b k}\right) .
\end{aligned}
$$

The variables appearing here $A^{i}, f_{i}, g^{i}(i=1,2,3,4), S, \theta^{n i},{ }^{a} \eta^{n i j},{ }^{a b} \zeta^{n i j k}(n, i, a, j, b, k=$ $1,2,3,4)$ depend only on $t^{1}, \ldots, t^{4}, u_{1}, \ldots, u_{4}$. More specifically,

the $A^{i}$ depend only on the $t$ 's and are the components of a conformal vector field in space time;

$f^{1}, \ldots, f^{4}$ depend only on $t$ and are a solution of $(2.1), f^{j, i}=\frac{\partial f^{j}}{\partial t_{i}}$ 
the $\theta, \eta, \zeta$ are alternating in $n i, n i j, n i j k$

respectively, and $\zeta$ is alternating in $a, b$;

$$
g^{1}, \ldots, g^{4} \text { depend only on } t \text { and } \partial g^{i} / \partial t^{i}=0
$$

Let $\varepsilon_{\Phi}$ be defined by (2.2) when $J^{i}$ is replaced by $\Phi^{i}$; let $\varepsilon_{\Psi}$ be defined by (2.2) with $J^{i}$ replaced by $\Psi^{i}$, and so forth. Then $\varepsilon=\varepsilon_{\Phi}+\cdots+\varepsilon_{N}$.

2.4. (Theorem). $\varepsilon_{\Phi}$ and $\varepsilon_{\Psi}$ are Noetherian forms.

$\varepsilon_{\Phi}$ is based on the infinitesimal conformal transformation

$$
A^{1} \frac{\partial}{\partial t^{1}}+\cdots+A^{4} \frac{\partial}{\partial t^{4}}
$$

$\varepsilon_{\Psi}$ is based on the infinitesimal translation in field-space:

$$
-f_{1} \frac{\partial}{\partial u_{1}}-\cdots-f_{4} \frac{\partial}{\partial u_{4}}
$$

$$
\text { If } \partial S / \partial u^{i}=0(i=1,2,3,4) \text { then } \varepsilon_{W} \text { is Noetherian, being }
$$

based on the gauge transformation

$$
-\frac{\partial S}{\partial t^{1}} \frac{\partial}{\partial u_{1}}-\cdots-\frac{\partial S}{\partial t^{4}} \frac{\partial}{\partial u_{4}} .
$$

If $\partial S / \partial u^{i}$ is not 0 for some $i$, then

$$
\varepsilon_{I}+\varepsilon_{W}+\varepsilon_{N}
$$

is not equivalent to any Noetherian form.

$$
\text { If } \varepsilon_{\Gamma}+\varepsilon_{N} \text { is equivalent to a Noetherian form } \omega \text {, }
$$

then $\omega$ is equivalent to 0 .

$$
\varepsilon_{I} \text { is exact, and hence equivalent to } 0 .
$$

The details of the definition of Noetherian forms are these. The Maxwell field has an action form ${ }^{1}$

$$
\alpha=-\frac{1}{2} p_{i j}\left(p^{i j}-p^{j i}\right) d^{1234}+d u_{i}\left[\left(p^{i 1}-p^{1 i}\right) d^{234}-+-\right],
$$

whose extremals are the motions.

Suppose $U$ is a vector field in the space $J^{1}(M, Q)$ such that the Lie derivative $\ell_{U} \alpha$ [3,p. 172] is exact $: \ell_{U} \alpha=d \psi$, where $\psi$ is some 3-form. Then Noether's theorem says $[2,5]$ that

$$
N(U)=U] \alpha-\psi
$$

is dynamic as defined above. Here $U\rfloor \alpha$ is the contraction of the vector $U$ with the 4-

1 From this point on, we omit the wedges $(\wedge)$ in writing differential forms Moreover $d t^{1} d t^{2} d t^{3} d t^{4}$ will be abbreviated to $d^{1234} d^{234}$ means $d t^{2} d t^{3} d t^{4}$ Finally, whenever $C^{1}, C^{2}, C^{3}, C^{4}$ are any four quantities, then $C^{1} d^{234}-+-$ stands for $C^{1} d^{234}-C^{2} d^{134}+C^{3} d^{124}-C^{4} d^{123}$ 
form $\alpha$. (If $\ell_{U} \alpha$ is exact then $U$ is called Hamiltonic [5] and sometimes the term Noetherian is applied only when $\ell_{U} \alpha=0$.)

We declare a 3-form $\varphi$ to be equivalent to another, $\psi$, if $\varphi-\psi$ is expressible as a $\operatorname{sum} \beta+\gamma$, where $\beta$ is exact and $\gamma$ is a form whose restriction to each motion is 0 . The 1 -forms

$$
X_{i}=d u_{i}-p_{i j} d t^{j}
$$

and the 2-forms

$$
d X_{i}=-d p_{i j} d t^{j}
$$

have this latter property of vanishing on all motions and so therefore do the 3-forms

$$
E^{i} X_{i}+Q^{i} d x_{i}
$$

where $E^{i}$ is any 2-form and $Q^{i}$, any 1 -form.

The converses of Theorems 2.3 and 2.4 are also true (and very easy to show). Thus if we select a function $S$ of $u$ and $t$ such that $\partial S / \partial u_{i} \neq 0$ for at least one $i$, then (2.34) gives us a dynamic form which is not Noetherian, nor even equivalent to a Noetherian form.

The rows (or columns) of the symmetric energy-momentum tensor $[4,7]$ form conserved currents of this kind. As shown in more detail (sect. 8 below) these wellknown dynamic forms are not equivalent to any Noetherian form.

The currents (2.31) (see also (2.41) constitute the very model of, or paradigm for, all Noetherian currents considered since 1921.

\section{Characteristic Properties of Dynamic Forms}

3.1 (Lemma). The 3-form (2.2) is dynamic if and only if

$$
\frac{\partial J^{i}}{\partial t^{i}}+p_{j i} \frac{\partial J^{i}}{\partial u_{j}}+A_{j k i} \frac{\partial J^{i}}{\partial p_{j k}}=0
$$

whenever (see (2.1))

$$
\left(A_{i j k}-A_{j i k}\right) g^{j k}=0 \text { and } A_{i j k}=A_{i k j}
$$

Proof. Suppose (2.2) is dynamic, and suppose that (3.12) holds. Let $t^{i}=a^{i}, u_{i}=b_{i}$, $p_{i j}=c_{i j}$ be a generic point of $J^{1}(M, Q)$.

For simplicity, suppose $a^{i}=0$. Let

$$
U_{i}=b_{i}+c_{i j} t^{j}+\frac{1}{2} A_{i j k} t^{j} t^{k}
$$

This is a solution of (2.1) if (3.3) hold; and then

$$
p_{i j}=c_{i j}+A_{i j k} t^{k}, u_{i}=b_{i}+c_{i j} t^{j}+\frac{1}{2} A_{i j k} t^{j} t^{k} .
$$

Therefore, by hypotheses, if we express the $u_{i}$ and $p_{i j}$ in $J^{i}$ by the expressions given by (3.5), then the divergence $\partial J^{i} / \partial t^{i}$ should be 0 . Using the chain rule, we obtain (3.2).

For the converse we must show that if (3.3) implies (3.2), then $\varepsilon$ is dynamic. This is obvious. 
Lemma. The differential form (2.2) is dynamic if and only if there exist variables $L^{k i j}$ and $M^{i}$ such that

$$
\frac{\partial J^{i}}{\partial t^{i}}+p_{j i} \frac{\partial J^{i}}{\partial u_{j}}+A_{j k i} \frac{\partial J^{i}}{\partial p_{j k}}=L^{k i j}\left(A_{k i j}-A_{k j i}\right)+M^{i} g^{j k}\left(A_{i j k}-A_{j i k}\right)
$$

is an identity in the $A$ 's.

In view of (3.1) this is an elementary proposition about polynomials of the first degree.

We may take all $A_{\imath j k}=0$ in the identity (3.6). Therefore, if (2.2) is dynamic then

$$
\frac{\partial J^{i}}{\partial t^{i}}+p_{j i} \frac{\partial J^{i}}{\partial u_{j}}=0
$$

By taking $A_{\lambda i j}=A_{\lambda j i}=1$, and $A_{\mu k m}=0$ for all other sets of indices, we obtain another relation: If $(2.2)$ is dynamic then there exist four variables $M^{i}$ such that

$$
\frac{\partial J^{i}}{\partial p_{\lambda j}}+\frac{\partial J^{j}}{\partial p_{\lambda i}}=-M^{i} g^{\lambda j}+2 M^{\lambda} g^{i j}-M^{j} g^{\lambda i}
$$

for $\lambda=1,2,3,4$.

Conversely, (3.7) and (3.8) together imply that (2.2) is dynamic. We begin by studying (3.8) by itself.

\section{The Implications of (3.8)}

The $M^{i}$ appearing in (3.8) will in general depend on all the coordinates in the jet bundle, but in (3.8) we are concerned only with the form of their dependence on the $p_{i j}$. Any variables arising which depend only on the $t$ 's and $u$ 's shall be called semiconstant in the following statement.

Theorem. The general solution to (3.8) is

$$
\begin{aligned}
M^{i}= & R^{i}+S_{k} p^{k i}-A_{k} p^{i k}, \\
J^{i}= & a^{i}+{ }^{a} \mu^{i j} p_{a j}+R_{j}\left(p^{j i}-p^{i j}\right)+\frac{1}{2} A^{i} p_{j k}\left(p^{j k}-p^{k j}\right)-A^{j} p_{k j}\left(p^{k i}-p^{i k}\right) \\
& +S^{k} p_{k j}\left(p^{j i}-p^{i j}\right)+{ }^{a b} \mu^{i j k} p_{a j} p_{b k}+{ }^{a b c} \mu^{i j k m} p_{a j} p_{b k} p_{c m}
\end{aligned}
$$

Here these coefficients are semi-constant. All indices range from 1 to 4 . The $\mu$ 's are alternating in the indices ijkm (to the extent that they have them) and similarly, alternating in the $a, b, c$. Indices are raised and lowered in the usual way.

The terms in (4.2) have been written in the order of their degree. Later the arrangement given by Theorem 2.3 will emerge as more natural.

We begin the proof by establishing (4.1).

Let us abbreviate

Clearly (3.4) makes

$$
\frac{\partial M^{j}}{\partial p_{\mu k}} \text { by } j \mu k
$$

$$
\frac{\partial^{2} J^{i}}{\partial p_{\mu k} \partial p_{\lambda j}}+\frac{\partial^{2} J^{i}}{\partial p_{\mu k} \partial p_{\lambda i}}=-g^{\lambda i}(j \mu k)+2 g^{i j}(\lambda \mu k)-g^{\lambda j}(i \mu k) .
$$


We permute to $i j k$ cyclically, twice, and add the three equations. The right hand side must then be symmetric in $\lambda$ and $\mu$, giving the equation

$$
\begin{aligned}
& 2 g^{i j}(\lambda \mu k-\mu \lambda k)+2 g^{j k}(\lambda \mu i-\mu \lambda i)+2 g^{k i}(\lambda \mu j-\mu \lambda j) \\
& \quad-g^{\lambda i}(j \mu k+k \mu j)-g^{\lambda j}(k \mu i+i \mu k)-g^{\lambda k}(i \mu j+j \mu i) \\
& \quad+g^{\mu i}(j \lambda k+k \lambda j)+g^{\mu j}(k \lambda i+i \lambda k)+g^{\mu k}(i \lambda j+j \lambda i)=0 .
\end{aligned}
$$

Let us agree that the metric $g^{i j}$ shall be diagonal.

We now establish a sequence of propositions (4.4-4.8).

$$
211=0, g^{11}(212+221)=g^{22}(111), 213+321=0 .
$$

To prove this, we take $i, j=1=\lambda, \mu=2$. Then

$$
\begin{aligned}
& 2 g^{11}(12 k-21 k)+2 g^{1 k}(121-211)+2 g^{k 1}(121-211)-g^{11}(12 k+k 21) \\
& \quad-g^{11}(k 21+12 k)-g^{1 k}(121+121)+g^{2 k}(111+111)=0 .
\end{aligned}
$$

We let $k=1$, and after some cancelling obtain $211=0$. For $k=2,3$ we obtain the other two relations.

$$
123=0
$$

For this we let $i, j, k=1$. The resulting equation, when divided by 6 , says $g^{11}(\lambda \mu 1$ $-\mu \lambda 1)-g \lambda^{1}(1 \mu 1)+g^{\mu 1}(1 \lambda 1)=0$. With $\lambda=2, \mu=3$, this says $231=321$ which by $(4.4)=-213$. So $M^{i j k}$ is alternating in $j k$ and symmetric in $i j$ when $i, j, k$ are distinct. So $123=213=-231=-321=312=-123$, and (4.5) holds.

$$
\frac{M^{i i j}}{g^{i i}} \text { is the same for all } i \neq j \text {, and so may be called }-A^{j} \text {. }
$$

To prove this, we take $\lambda=j=1, i=\mu=2, k=3$, obtaining $-g^{11}(322+223)$ $+g^{22}(113+311)=0$. From (4.4) we deduce 322 and 311 are 0 , whence

$$
\frac{223}{g^{22}}=\frac{113}{g^{11}}
$$

This establishes (4.6).

$$
\frac{M^{i j i}}{g^{i i}} \text { is the same for all } i \neq j \text { and so may be called } S^{j} \text {. }
$$

For this we let $i=j=1, k=\mu=2, \lambda=3$. Then $2 g^{11}(322-232)+g^{22}(131+131)$ $=0$. Observing that $322=0(4.3)$, we get

$$
\frac{131}{g^{11}}=\frac{232}{g^{22}}
$$

and (4.7) is established.

We now assert that

$$
M^{i \lambda j}=g^{i j} S^{\lambda}-g^{i \lambda} A^{j}
$$

Proof. From (4.4) we have $g^{22}(111)=g^{11}(212+221)$. This is exactly (4.8) for $i=j$ 
$=\lambda=1$. For $i=1, j=2$ in (4.6) we get (4.8) for $i=\lambda=1, j=2$. For $i=j=1, \lambda=2$, (4.8) reduces to (4.6). For $M^{122},(4.8)$ says $122=0$. We also know $M^{123}=0$. All other cases are equivalent to one of these five.

Now differentiate (4.8) with respect to $p_{\mu k}$ :

$$
\frac{\partial^{2} M^{i}}{\partial p_{\lambda j} \partial p_{\mu k}}=g^{i j} \frac{\partial S^{\lambda}}{\partial p_{\mu k}}-g^{i \lambda} \frac{\partial A^{j}}{\partial p_{\mu k}} .
$$

The right hand side therefore also equals

$$
g^{i k} \frac{\partial S^{\mu}}{\partial p_{\lambda j}}-g^{i \mu} \frac{\partial A^{k}}{\partial p_{\lambda j}} .
$$

Let $j, k, \mu$ be any indices. We can select $\lambda$ distinct from these. Let $i=\lambda$. So $-g^{i i} \partial A^{j} / \partial p_{\mu k}=0$. Thus $A^{j}$ is a semi-constant. The same can be seen for $S^{\lambda}$. Thus we have (4.1) where $R^{i}$ also is a semi-constant.

\section{The Proof of (4.2)}

Proposition. Let

$$
L^{k}=p_{m n}\left(p^{n k}-p^{k m}\right) S^{n}+\frac{1}{2} p_{m n}\left(p^{m n}-p^{n m}\right) A^{k}+\left(p^{m k}-p^{k m}\right)\left(R_{m}-p_{m n} A^{n}\right) .
$$

Then $L^{k}$ satisfies (3.8) with the $M^{i}$ as in (4.1), that is $\partial L^{i} / \partial p_{\lambda j}+\partial L^{j} / \partial p_{\lambda i}=$ right hand side of (3.8).

This is easily verified, and we omit the proof.

The hypothesis of (4.2) is that the $J^{i}$ are given. From (3.4) we get the $M^{\prime}$ 's and from (4.1), the $A, R$, and $S$. Thus the $J^{i}$ lead to the $L^{i}$ and to the $Z^{i}=J^{i}-L^{i}$.

Obviously

$$
\frac{\partial Z^{i}}{\partial p_{\lambda j}}+\frac{\partial Z^{j}}{\partial p_{\lambda i}}=0 .
$$

These equations say that for each $\lambda$, the $Z^{i}$ are the components of an infinitesimal metric-preserving, (thus in our case Poincaré) vector field in an $\mathbb{R}^{4}$ in which $p_{\lambda 1}, \ldots, p_{\lambda 4}$ are the cartesian coordinates. Thus $Z^{i}$ has the form

$$
a_{(\lambda)}^{i}+\mu_{(\lambda)}^{i k} p_{\lambda k} \quad(\text { no sum on } \lambda),
$$

where $a_{(\lambda)}^{i}$ and $\mu_{(\lambda)}^{i j}=-\mu_{(\lambda)}^{j i}$ are independent of $p_{\lambda 1}, \ldots, p_{\lambda 4}$.

Let us take $\lambda=1$ and then $Z^{i}=a_{(1)}^{i}+\mu_{(1)}^{i k} p_{1 k}$. It is easily seen that $a_{(1)}^{i}$ and $\mu_{(1)}^{i k}$ (for a fixed $k$ ) again satisfy (5.1) for $\lambda=2,3$, or 4; and thus have the form(5.2). Making two more applications of this idea one obtains

$$
Z^{i}=a^{i}+{ }^{a} \mu^{i j} p_{a j}+{ }^{a b} \mu^{i j k} p_{a j} p_{b k}+{ }^{a b c} \mu^{i j k m} p_{a j} p_{b k} p_{c m} .
$$

Here the $\mu$ are certainly alternating in the $i, j, k, m$. There is also summation over the $a, b, c$ but if the construction is performed in the natural order we have $a<b<c$ in the last term and $a<b$ in the previous one. Let us define ${ }^{a b} \mu^{i j k}$ for $a \geqq b$ by requiring it to be alternating in the indices $a, b$, and similarly for the last term. After absorbing the factorials, we have $Z^{i}$ in the form above with alternation in the $a, b, c$, as well. The 
$a^{i}$ and the $\mu$ 's are semi-constants (i.e. independent of the $p_{i j}$.) Thus $J^{i}$ has the very form displayed in (4.2), and the theorem is proved.

We next examine what further restrictions are imposed on the coefficients in (4.2) by the other necessary condition, (3.7).

\section{The Consequences of (3.7), and Proof of Theorem 2.3}

When we insert (4.2) into (3.7), we get a polynomial of the fourth degree in the p's, which has to vanish. Let $J^{n}={ }^{a b c} \mu^{n i j k} p_{a i} p_{b j} p_{c k}+$ lower degree. Write (3.7) in the form

$$
\frac{\partial J^{n}}{\partial t^{n}}+\frac{\partial J^{n}}{\partial u_{m}} p_{m n}=0 \text {. }
$$

Then the fourth degree terms must satisfy

$$
\frac{\partial^{a b c} \mu^{n i j k}}{\partial u_{m}} p_{a i} p_{b j} p_{c k} p_{m n}=0 .
$$

Denote this coefficient by [mabc;nijk]. Let $\sigma$ be any permutation on four letters. Let $\sigma(m a b c)$ be the image of $m a b c$ under $\sigma$. Then $\sum_{\sigma}[\sigma(m a b c) ; \sigma(n i j k)]=0$. We know $\mu$ is alternating in nijk so, summing over 24 permutations $\sigma, \Sigma \operatorname{sgn}(\sigma)[\sigma(m a b c), n i j k]=0$. Fix nijk and denote ${ }^{a b c} \mu^{n i j k}$ by ${ }^{a b c} \mu$. Since $\mu$ is alternating in $a b c$ we obtain

$$
{ }^{m a b c} \mu-{ }^{a m b c} \mu+{ }^{b m a c} \mu-{ }^{c m a b} \mu=0,
$$

or

$$
\frac{\partial^{a b c} \mu}{\partial u_{m}}-\frac{\partial^{m b c} \mu}{\partial u_{a}}+\frac{\partial^{m a c} \mu}{\partial u_{b}}-\frac{\partial^{m a b} \mu}{\partial u_{c}}=0 .
$$

This says that the (tensor valued) 3-form

is closed, whence exact, so that

$$
{ }^{a b c} \mu d u_{a} d u_{b} d u_{c}
$$

$$
{ }^{a b c} \mu=\frac{\partial^{b c} \beta}{\partial u^{a}}-\frac{\partial^{a c} \beta}{\partial u^{b}}+\frac{\partial^{a b} \beta}{\partial u^{c}}
$$

Here ${ }^{a b} \beta$ has components ${ }^{a b} \beta^{n i j k}$. This system is alternating in $a b$, and alternating $n i j k$. Let us write

$$
{ }^{c / a b} \beta \text { for } \frac{\partial^{a b} \beta}{\partial u_{c}}
$$

and let us write

$$
{ }^{c / a b} \beta_{m}^{n i j k} \text { for } \frac{\partial^{c / a b} \beta^{n i j k}}{\partial t^{m}} .
$$

Now we look at the cubic terms of equation (3.7), mentally first replacing the $i$ and $j$ there by $n$ and $r$. For $J^{n}$ we consult (4.2). Then (3.7) produces

$$
\begin{gathered}
\left({ }^{a / b c} \beta_{n}^{n i j k}-{ }^{b / a c} \beta_{n}^{n i j k}+{ }^{c / a b} \beta_{n}^{n i j k}\right) p_{a i} p_{b j} p_{c k}+p_{r n} \frac{\partial}{\partial u_{r}}\left[\frac{1}{2} A^{n} p_{j k}\left(p^{j k}-p^{k j}\right)\right. \\
\left.-A^{j} p_{k j}\left(p^{k n}-p^{n k}\right)+S^{k} p_{k j}\left(p^{j n}-p^{n j}\right)+{ }^{a b} \mu^{n j k} p_{a j} p_{b k}\right]=0 .
\end{gathered}
$$


The three terms containing the $\beta$ can be written as

where

$$
p_{r k} \frac{\partial}{\partial u_{r}}{ }^{a b} \gamma^{i j k} p_{a i} p_{b j}
$$

$$
{ }^{a b} \gamma^{i j k}=3^{a b} \beta_{n}^{n i j k}
$$

Therefore

$$
p_{r n} \frac{\partial}{\partial u_{r}}\left[{ }^{a b} \gamma^{i j n} p_{a i} p_{b j}+{ }^{a b} \mu^{i j n} p_{a i} p_{b j}+\cdots\right]=0,
$$

where the dots represent the $A$ and $S$ terms in (6.2).

Let $\gamma+\mu$ be denoted by $\sigma$. Then the entire equation is

$$
\begin{aligned}
& { }^{c / a b} \sigma^{i j k} p_{a i} p_{b j} p_{c k}+\frac{1}{2} A^{n r} p_{r n} p_{j k}\left(p^{j k}-p^{k j}\right)-A^{j r} p_{r n} p_{k j}\left(p^{k n}-p^{n k}\right) \\
& \quad+S^{k r} p_{r n} p_{k j}\left(p^{j n}-p^{n j}\right)=0 .
\end{aligned}
$$

Here the second index on $A$ and $S$ indicates the derivative with respect to $u_{r}$. In this equation let $p_{i j}=y_{j}$ when $j=1$, and let $p_{i j}=0$ when $j \neq 1$. Then the $\sigma$ terms vanish and in fact the equation simplifies to

$$
A^{n 1} y_{n}\left(-\frac{1}{2} p^{1 j} y_{j}+y_{1} p^{11}-\frac{1}{2} y_{j} p^{j 1}\right)=0 .
$$

This implies that $\partial A^{n} / \partial u_{m}=0$ for all $m$ and $n$.

Now we go back to (6.3), erase the $A$ terms, and let $p_{1 i}=x_{i}, p_{2 i}=y_{i}$ and all other $p ' s=0$. Again the $\sigma$ terms vanish, and the equation reduces to $\left(S^{12}-S^{21}\right) x_{i} y_{m} \times$ $\left(p^{m i}-p^{i m}\right)=0$. Therefore there is a function $S$ of $t$ and $u$ such that $S^{k}=\partial S / \partial u_{k}$.

The fact that $S^{k r}=S^{r k}$ makes the $S$ terms disappear in (6.3), leaving only ${ }^{c / a b} \sigma^{i j k} p_{a i} p_{b j} p_{c k}=0$. From this we deduce that

$$
{\frac{\partial}{\partial u_{c}}}^{a b} \sigma^{i j k}+{\frac{\partial}{\partial u_{a}}}^{b c} \sigma^{i j k}+{\frac{\partial}{\partial u_{b}}}^{c a} \sigma^{i j k}=0,
$$

using the fact that $\sigma$ is alternating in $i j k$.

This shows that (for each $i j k$ ) $\sigma={ }^{a b} \sigma^{i j k} d u_{a} d u_{b}$ is exact, whence there is a form $\tau={ }^{a} \eta^{i j k} d u_{a}$ such that $\sigma=d \tau$. In coordinate form.

$$
{ }^{a b} \sigma^{i j k}=\frac{\partial}{\partial u_{a}}\left({ }^{b} \eta^{i j k}\right)-\frac{\partial}{\partial u_{b}}\left({ }^{a} \eta^{i j k}\right)={ }^{a b} \mu^{i j k}+3^{a b} \beta_{n}^{n i j k} .
$$

We mentally insert the formula which this provides for $\mu$ into (4.2) and write down the second degree terms of Eq. (3.3). The result is

$$
\begin{aligned}
& \left({ }^{a / b} \eta_{i}^{i j k}-{ }^{b / a} \eta_{i}^{i j k}\right) p_{a j} p_{b k}+{ }^{b / a} \mu^{i j} p_{b i} p_{a j} \\
& \quad+S_{i}^{n} p_{n m}\left(p^{m i}-p^{i m}\right)-A_{i}^{n} p_{m n}\left(p^{m i}-p^{i m}\right) \\
& \quad+R_{i}^{n} p_{n m}\left(p^{i m}-p^{m i}\right)+\frac{1}{2} A_{i}^{i} p_{m n}\left(p^{m n}-p^{n m}\right)=0 .
\end{aligned}
$$

There is no $\beta$ here because the sum ${ }^{a b} \beta_{n i}^{n i j k}$ is zero. Here $S_{i}^{n}=\partial S^{n} / \partial t^{i}$, where $S^{n}$ $=\partial S / \partial u_{n} ; A_{i}^{n}=\partial A^{n} / \partial t^{i}$; but $R_{i}^{n}=\partial R_{i} / \partial u_{n}$. The $R$ and $S$ terms can be combined into $\left(S_{i}^{n}-R_{i}^{n}\right) p_{n m}\left(p^{m i}-p^{i m}\right)$. Let $S_{i}^{n}-R_{i}^{n}$ be abbreviated to $D_{i}^{n}$. Let $p_{n m}=x_{m}$ when $n=1$, and 0 otherwise. The $\eta$ and $\mu$ terms vanish with such a choice of the $p$ 's, and the 
equation reduces to

$$
D_{i}^{1} x_{1} p^{1 i}-D_{1}^{1} x_{m} p^{1 m}-A_{i}^{n} x_{n} p^{1 i}+A_{1}^{n} x_{n} p^{11}+\frac{1}{2} A_{i}^{i}\left(x_{n} p^{1 n}-x_{1} p^{11}\right)=0 .
$$

Examining the coefficient of $x_{1} x_{2}$ we find that $A_{2}^{1}-D_{2}^{1}=0$. The coefficient of $x_{2} x_{2}$ tells us that $D_{1}^{1}=-A_{2}^{2}+\frac{1}{2} A_{i}^{i}$. This relation implies eleven others which force $A_{1}^{1}=A_{2}^{2}=A_{3}^{3}=A_{4}^{4}$, which value we may call $A$, and $D_{1}^{1}=D_{2}^{2}=D_{3}^{3}=D_{4}^{4}=D$. It follows at once that $D=A$.

From $A_{2}^{1}=D_{2}^{1}$ and $A_{1}^{1}=D_{1}^{1}$ we deduce that $A_{i}^{j}=D_{i}^{j}$, which says that

This suggests letting

$$
\frac{\partial A^{j}}{\partial t^{i}}+\frac{\partial R_{i}}{\partial u_{j}}=\frac{\partial^{2} S}{\partial t^{i} \partial u_{j}}
$$

$$
f_{i}=\frac{\partial S}{\partial t^{i}}-R_{i}-\frac{\partial A^{j}}{\partial t^{i}} u_{j}
$$

Then

$$
\frac{\partial f_{i}}{\partial u_{j}}=\frac{\partial^{2} S}{\partial t^{i} \partial u_{j}}-\frac{\partial R_{i}}{\partial u_{j}}-\frac{\partial A^{j}}{\partial t^{i}}=0 .
$$

Examining the coefficient of $x_{2} x_{3}$ we find that $\partial A^{2} / \partial t_{3}+\partial A^{3} / \partial t_{2}=0$. Since $A_{1}^{1}=\cdots=A_{4}^{4}=A$ we can assert that $\partial A^{i} / \partial t_{j}+\partial A^{j} / \partial t_{i}=2 g^{i j} A$ for all $i$ and $j$ (Recall that we chose $g$ to be diagonal.) We have shown [1] that this is a necessary and sufficient condition for the vector field $A^{i}$ to be conformal in space time in the appropriate Minkowski sense, so that there are constants $a^{i}, m^{i j}\left(=-m^{j i}\right), \lambda, b^{i}$ such that

$$
A^{i}=\frac{1}{2} a^{i} t_{j} t^{j}-t^{i} a_{j} t^{j}+m^{i j} t_{j}+\lambda t^{i}+b^{i} .
$$

A routine calculation shows that this formula, together with the $S_{i}^{j}-R_{i}^{j}=A_{i}^{j}$ makes all the $S, A$, and $T$ terms disappear from our quadratic equation, leaving us just the problem of studying the equation

$$
\left({ }^{a / b} \eta_{i}^{i j k}-{ }^{b / a} \eta_{i}^{i j k}+{ }^{b / a} \mu^{k j}\right) p_{a j} p_{b k}=0 .
$$

Just as earlier, this says that a certain tensor valued differential form in $d u$ is exact. This time it is a 1 -form, and the result is that for each $j$ and $m$

$$
{ }^{a} \mu^{j m}=-2^{a} \eta_{i}^{i j m}+\frac{\partial \theta^{j m}}{\partial u_{a}} .
$$

This $\theta$ is alternating in $j m$.

Now we come to the linear terms in Eq. (3.7) or rather (6.1). Looking at (4.2) and taking (6.5) into account, we have

$$
\frac{\partial}{\partial t^{n}}\left(-2^{a} \eta_{i}^{i n j}+\frac{\partial \theta^{n j}}{\partial u_{a}}\right) p_{a j}+\frac{\partial R_{j}}{\partial t^{n}}\left(p^{j n}-p^{n j}\right)+p_{m n} \frac{\partial a^{n}}{\partial u_{m}}=0
$$

We note $\eta_{i n}^{i n j}=0$, and take the coefficient of $p_{h k}$,

$$
\frac{\partial \theta_{n}^{n k}}{\partial u_{h}}+\frac{\partial R_{i}}{\partial t^{n}}\left(g^{i h} g^{n k}-g^{n h} g^{i k}\right)+\frac{\partial a^{k}}{\partial u_{h}}=0 .
$$


From (6.4) we obtain

$$
\frac{\partial R_{i}}{\partial t^{n}}=-\frac{\partial f_{i}}{\partial t^{n}}+\frac{\partial^{2} S}{\partial t^{n} \partial t^{i}}-\frac{\partial^{2} A^{j}}{\partial t^{n} \partial t^{i}} u_{j},
$$

and so

$$
\frac{\partial}{\partial u_{h}}\left(\theta_{n}^{n k}+a^{k}\right)-\frac{\partial f^{h}}{\partial t_{k}}+\frac{\partial f^{k}}{\partial t_{h}}=0
$$

Therefore

$$
\theta_{n}^{n k}+a^{k}=\left(\frac{\partial f^{h}}{\partial t_{k}}-\frac{\partial f^{k}}{\partial t_{h}}\right) u_{h}+g^{k},
$$

where $g^{k}$ depends only on $t$.

We arrive at the $0^{\text {th }}$ degree terms (in $p$ ) of (6.1), which say no more than $\partial a^{m} / \partial t^{m}=0$. Combining this with (6.6) yields

$$
\theta_{n k}^{n k}=\frac{\partial}{\partial t^{k}}\left(\frac{\partial f^{h}}{\partial t_{k}}-\frac{\partial f^{k}}{\partial t_{h}}\right) u_{h}+\frac{\partial g^{k}}{\partial t^{k}} .
$$

Therefore $\partial g^{k} / \partial t^{k}=0$ (as in (2.39)) and

which is precisely (2.37).

$$
\frac{\partial}{\partial t^{k}}\left(\frac{\partial f^{k}}{\partial t_{k}}-\frac{\partial f^{h}}{\partial t^{k}}\right)=0
$$

Let us put all our findings into (4.2). The result is

$$
\begin{aligned}
J^{i}= & g^{i}+\left(f^{h, i}-f^{i, h}\right) u_{h}-\theta_{n}^{n i}+\left(-2^{a} \eta_{n}^{n i j}+\frac{\partial \theta^{i j}}{\partial u_{a}}\right) p_{a j} \\
& +\left(\frac{\partial S}{\partial t^{j}}-f_{j}-\frac{\partial A^{k}}{\partial t^{j}} u_{k}\right)\left(p^{j i}-p^{i j}\right)+\frac{1}{2} A^{i} p_{j k}\left(p^{j k}-p^{k j}\right) \\
& -A^{j} p_{k j}\left(p^{k i}-p^{i k}\right)+S^{k} p_{k j}\left(p^{j i}-p^{i j}\right) \\
& +\left(-3^{a b} \beta_{n}^{n i j k}-a / b \eta^{i j k}+{ }^{b / a} \eta^{i j k}\right) p_{a j} p_{b k} \\
& +\left({ }^{a / b c} \beta^{i j k m}-{ }^{b / a c} \beta^{i j k m}+{ }^{c / a b} \beta^{i j k m}\right) p_{a j} p_{b k} p_{c m} .
\end{aligned}
$$

The reader can pick out here precisely the terms given in (2.31), (2.32), (2.33), and (2.34). The remainder gives (2.35) when we replace $-\theta$ by $\theta,-2 \eta$ by $\eta$, and $-3 \beta$ by $\zeta$. After this change of notation, (2.3) has been established.

\section{Noetherian Forms. Proof of (2.44) and (2.45)}

To use definition (2.6), one must find a $U$ such that $\ell_{U} \alpha$ is exact. To prove (2.41) for example, we have to find such a $U$; but we don't mean to assert that

$$
A^{1} \frac{\partial}{\partial t^{1}}+\cdots+A^{4} \frac{\partial}{\partial t^{4}}
$$

can be taken as $U$. We proceed to explain what we mean by "based on the infinitesimal conformal transformation (7.1)", in statement (2.41).

As explained by Noether and Bessel-Hagen [2], one must use (7.1) to produce a vector field $U$ in the jet bundle. Knowing the field involved, one must note how the 
field components $u_{i}$ and their derivatives change when the coordinates change (infinitesimally). The discussion by Jauch $[7, p .218]$ is exemplary, except that he discusses only Lorentz transformations.

Quite abstractly, let (7.1) induce the infinitesimal transformation $\delta u_{\lambda}=B_{\lambda}$ for the field. Then this will induce the transformation

for the derivatives.

$$
\delta p_{\lambda k}=\frac{\partial B_{\lambda}}{\partial t^{k}}+\frac{\partial B_{\lambda}}{\partial u_{\mu}} p_{\mu k}-\frac{\partial A^{j}}{\partial t^{k}} p_{\lambda j}
$$

In the Maxwellian context, one has

$$
B_{\lambda}=-\frac{\partial A^{i}}{\partial t^{\lambda}} u_{i}
$$

(Please keep in mind that $u_{1}, \ldots, u_{4}$ is the covector potential, and the $A$ 's are just the components of (7.1). Thus

$$
\delta p_{\lambda k}=-\frac{\partial^{2} A^{i}}{\partial t^{k} \partial t^{\lambda}} u_{i}-\frac{\partial A^{j}}{\partial t^{\lambda}} p_{j k}-\frac{\partial A^{j}}{\partial t^{k}} p_{\lambda j} \equiv C_{\lambda k}
$$

The desired vector field is

$$
U=A^{i} \frac{\partial}{\partial t^{i}}+B_{\lambda} \frac{\partial}{\partial u_{\lambda}}+C_{\lambda k} \frac{\partial}{\partial p_{\lambda k}} .
$$

The next question is whether $\ell_{U} \alpha$ is exact. This is the point of [2]: $\ell_{U} \alpha=0$. Knowing this, we can assert that $N(U)=U\rfloor \alpha$ is a dynamic form (and the $C_{\lambda k}$ having served their purpose, may be forgotten.) Computation shows that

$$
U\rfloor \alpha=\Phi^{1} d^{234}-\Phi^{2} d^{134}+\Phi^{3} d^{124}-\Phi^{4} d^{123}+\gamma,
$$

where the $\Phi^{i}$ are as in (2.31), and $\gamma$ is a form like (2.9). That is what we mean by "based on," so (2.41) is proved.

The computation is best done as follows. Compute $U\rfloor \alpha$, and then replace $d u_{i}$ by $p_{i j} d t^{j}$. Then the result is $\Phi^{1} d^{234}-+-$. The $\gamma$ term comes from this replacement.

We now address ourselves to (2.42). Here we have already committed ourselves to two sets of components: $\delta t^{i}=0, \delta u_{\lambda}=-f_{\lambda}$; and so, by (7.2),

This makes

$$
\delta p_{\lambda k}=-\frac{\partial f_{\lambda}}{\partial t^{k}} \equiv-f_{\lambda k}
$$

$$
-U=f_{\lambda} \frac{\partial}{\partial u_{\lambda}}+f_{\lambda k} \frac{\partial}{\partial p_{\lambda k}} .
$$

Referring to (2.5) we deduce [3,p.172] that

$$
\begin{aligned}
-\ell_{U} \alpha= & -\frac{1}{2}\left[f_{i j}\left(p^{i j}-p^{j i}\right)+p_{i j}\left(f^{i j}-f^{j i}\right)\right] d^{1234} \\
& +d f_{i}\left[\left(p^{i 1}-p^{1 i}\right) d^{234}-+-\right]+d u_{i}\left[\left(f^{i 1}-f^{1 i}\right) d^{234}-+-\right] \\
= & -\left[f_{i j}\left(p^{i j}-p^{j i}\right)\right] d^{1234} \\
& +f_{i 1}\left(p^{i 1}-p^{1 i}\right) d^{1234}+f_{i 2}\left(p^{i 2}-p^{2 i}\right) d^{1234}+\cdots \\
& +d u_{i}\left[\left(f^{i 1}-f^{1 i}\right) d^{234}-+-\right] \\
= & d u_{i}\left[\left(f^{i 1}-f^{1 i}\right) d^{234}-+-\right] .
\end{aligned}
$$




$$
\begin{aligned}
& \text { Let }-\psi=u_{i}\left[\left(f^{i 1}-f^{1 i}\right) d^{234}-+-\right] \text {. Then } \\
& -d \psi= \\
& d u_{i}\left[\left(f^{i 1}-f^{1 i}\right) d^{234}-+-\right]+u_{i}\left[\left(f^{i 1}-f^{1 i}\right)_{1} d^{1234}\right. \\
& \left.+\left(f^{i 2}-f^{2 i}\right)_{2} d^{1234}+\cdots\right]=-\ell_{U} \alpha,
\end{aligned}
$$

because the subsequent terms vanish, on account of Maxwell's equations (2.1) for the $f^{i}$. Thus a Noetherian current is in sight. We need

and so $N(U)$ is

$$
U\rfloor \alpha=-f_{i}\left[\left(p^{i 1}-p^{1 i}\right) d^{234}-+-\right],
$$

$$
\begin{aligned}
U] \alpha-\psi & =\left[u_{i}\left(f^{i 1}-f^{1 i}\right)-f_{i}\left(p^{i 1}-p^{1 i}\right)\right] d^{234}-+- \\
& =\psi^{1} d^{234}-+-,
\end{aligned}
$$

where $\psi^{i}$ is as in (2.32). Thus (2.42) is proved.

We now consider (2.43). If $\partial S / \partial u=0$, then (2.34) is a special case of (2.32) because $S^{j, i}=S^{i, j}$. Hence our proof of (2.42) provides a proof of (2.43). Incidentally, $\ell_{U} \alpha$ is 0 .

The dynamic form $\varepsilon_{\Gamma}$, to wit $g^{1} d^{234}-+-$ is exact because

$$
d \varepsilon_{\Gamma}=g_{1}^{1} d^{1234}+\cdots=g_{i}^{i} d^{1234}=0
$$

so $\varepsilon_{\Gamma}$ is equivalent to the 0 form. Hence it remains to show that $\varepsilon_{W}+\varepsilon_{N}$ cannot be equivalent to a Noether form if $\partial S / \partial u \neq 0$.

Lemma. Suppose $\varepsilon$ is equivalent to a Noetherian form. Then there is a vector field $U$ and a form $\zeta$ which is 0 on all motions such that

$$
d \varepsilon+U\rfloor d \alpha+d \zeta=0
$$

Proof. If $\varepsilon$ is equivalent to a Noether form $N(U)$ then $\varepsilon=N(U)-\zeta+\mathrm{d} \theta$. Here $N(U)=U\rfloor \alpha-\psi$, where $\ell_{U} \alpha=d \psi$. So

$$
\begin{aligned}
d \varepsilon & =d(U\rfloor \alpha)-d \psi-d \zeta \\
& =d(U\rfloor \alpha)-\ell_{U} \alpha-d \zeta \\
& =d(U\rfloor \alpha)-d(U] \alpha)-U] d \alpha-d \zeta,
\end{aligned}
$$

which proves (7.3). Here we used [3,p. 172].

We need appropriate formulas for the ingredients of (7.3).

Lemma. Let $\varepsilon=J^{1} d^{234}-+-$ be dynamic. Then

$$
d \varepsilon=d_{p} J^{1} d^{234}-+-+X_{j}\left(\frac{\partial J^{1}}{\partial u_{j}} d^{234}-+-\right) .
$$

For the proof of the lemma,

$$
d \varepsilon=\frac{\partial J^{i}}{\partial t^{i}} d^{1234}+d_{p} J^{1} d^{234}-+-+\frac{\partial J^{1}}{\partial u_{j}} d u_{j} d^{234}-+-
$$

where $d_{p}$ means take $d$ but regard only the $p$ 's as variable. By (2.7),

$$
d \varepsilon=d_{p} J^{1} d^{234}-+-+\left(\frac{\partial J^{i}}{\partial t^{i}}+p_{j i} \frac{\partial J^{i}}{\partial u_{j}}\right) d^{1234}+X_{j}\left(\frac{\partial J^{1}}{\partial u_{j}} d^{234}-+-\right) \text {. }
$$


Reference to (3.7) shows that the desired result holds.

We deduce from (2.5) that

$$
d \alpha=-\left(p^{i j}-p^{j i}\right) d p_{i j} d^{1234}-d u_{i}\left[\left(d p^{1 i}-d p^{i 1}\right) d^{234}-+-\right] .
$$

We take an arbitrary vector field

and contract it with $d \alpha$ :

$$
U=A^{i} \frac{\partial}{\partial t^{i}}+B_{i} \frac{\partial}{\partial u_{i}}+C_{i j} \frac{\partial}{\partial p_{i j}}
$$

$$
\begin{aligned}
U] d \alpha= & \left(A^{k} p_{i k}-B_{i}\right)\left[\left(d p^{i 1}-d p^{1 i}\right) d^{234}-+-\right] \\
& +X_{i}\left[\left(C^{i 1}-C^{1 i}\right)\left(d^{234}-+-\right)\right. \\
& -\left(d p^{i 1}-d p^{1 i}\right)\left(A^{2} d^{34}-A^{3} d^{24}+A^{4} d^{23}\right) \\
& +\left(d p^{i 2}-d p^{2 i}\right)\left(A^{1} d^{34}-A^{3} d^{14}+A^{4} d^{13}\right) \\
& -\left(d p^{i 3}-d p^{3 i}\right)\left(A^{1} d^{24}-++\right) \\
& \left.+\left(d p^{i 4}-d p^{4 i}\right)\left(A^{1} d^{23}-+\right)\right] .
\end{aligned}
$$

Here we replaced $d u_{i}$ by use of (2.7).

In view of (2.9), we write $\zeta=-X_{i} E^{i}+d p_{i j} d t^{j} a^{i k l} d p_{k l}$, where

$$
\begin{aligned}
E^{i}= & F^{i j k h m} d p_{j k} d p_{h m}+G_{m}^{i j k} d p_{j k} d t^{m}+H_{j k}^{i} d t^{j} d t^{k} \\
& +I^{i j k} X_{j} X_{k}+K_{k}^{i j} d t^{k} X_{j}+L^{i j k m} d p_{j k} X_{m}
\end{aligned}
$$

In mentally forming $d E$ one should keep in mind the rule (for any function of the $p, u, t)$

where

$$
d F=\frac{\partial F}{\partial p_{i j}} d p_{i j}+\frac{\partial F}{\partial u_{i}} X_{i}+\left(D_{i} F\right) d t^{i}
$$

$$
D_{i}=\frac{\partial}{\partial t^{i}}+p_{j i} \frac{\partial}{\partial u_{j}} .
$$

\section{Lemma.}

$$
G_{m}^{i j k}=D_{m} a^{i j k}+\gamma^{i j} \delta_{m}^{k}, \gamma^{i j}=-\gamma^{j i}
$$

Proof. We look at the pptt terms in the sum (7.3). By this we mean terms with $d p_{j k} d p_{h i} d t^{m} d t^{n}$ when the $d p_{j k}, d t^{i}, X_{m}$ are chosen as a basis. Now $d \varepsilon$ has no such terms, nor has $U\rfloor d \alpha$, but

$$
d \zeta=-d X_{i} E^{i}+X_{i} d E^{i}+d p_{i j} d t^{j} d a^{i k h} d p_{k h}=X_{i} d E^{i}+d p_{i j} d t^{j}\left(E^{i}+d a^{i k h} d p_{k h}\right)
$$

does, namely

$$
\Gamma_{m}^{i n k} d p_{i j} d t^{j} d p_{n k} d t^{m}
$$

where $\Gamma_{n}^{i n k}=G_{m}^{i n k}+D_{m} a^{i n k}$. Thus (7.5) can be equated to 0 . Let us take the coefficient of $d^{34}$ here: $\Gamma_{4}^{i n k} d p_{i 3} d p_{n k}-\Gamma_{3}^{i n k} d p_{i 4} d p_{n k}=0$. Contract this with $\partial / \partial p_{m 1}$. This gives $-\Gamma_{4}^{i m 1} d p_{i 3}+\Gamma_{3}^{i m 1} d p_{i 4}=0$. Clearly $\Gamma_{k}^{i m j}=0$ if $j \neq k$.

Now we go back and contract with $\partial / \partial p_{m 4}$ :

$$
-\Gamma_{4}^{i m 4} d p_{i 3}+\Gamma_{3}^{i m 4} d p_{i 4}-\Gamma_{3}^{m n k} d p_{n k}=0 .
$$


Now contract this with $\partial / \partial p_{j 3}$ :

$$
-\Gamma_{4}^{j m 4}-\Gamma_{3}^{m j 3}=0, \quad \text { or } \Gamma_{4}^{j k 4}=-\Gamma_{3}^{k j 3} .
$$

This is therefore true for other indices, so $\Gamma_{4}^{j k 4}=-\Gamma_{3}^{k j 3}=+\Gamma_{2}^{j k 2}$. One concludes that $\Gamma_{n}^{j k m}=\gamma^{j k} \delta_{n}^{m}$. Insertion into (7.5) (equated to 0) shows that $\gamma^{k j}=-\gamma^{j k}$. Thus (7.4) is proved.

\section{Lemma}

$$
2 H_{34}^{1}=\frac{\partial J^{2}}{\partial p_{11}}, 2 H_{34}^{3}=\frac{\partial J^{2}}{\partial p_{31}} .
$$

Proof. We look at the pttt terms in (7.3). There $d \varepsilon$ provides $\left.d_{p} J^{1} d^{234}-+-, U\right\rfloor d \alpha$ provides $\left(A^{k} p_{i k}-B_{i}\right)\left(d p^{i 1}-d p^{1 i}\right) d^{234}-+-, d \zeta$ provides $d p_{i j} d t^{j} H_{k h}^{i} d t^{k} d t^{h}$. Select out the $d p_{11} d^{134}$ terms:

$$
-\frac{\partial J^{2}}{\partial p_{11}}+2 H_{34}^{1}=0
$$

Next, select out the $d p_{31} d^{134}$ terms. The $\left.U\right\rfloor d \alpha$ has none. So $-\partial J^{2} / \partial p_{31}+2 H_{34}^{3}=0$. End of proof.

We now prove (2.44). We may assume $J^{i}=N^{i}+W^{i}$. Let us look at the $X_{i} p d^{34}$ terms in (7.3). The $d \varepsilon$ has none, $U\rfloor d \alpha$ provides.

$-\left(d p^{i 1}-d p^{1 i}\right) A^{2}+\left(d p^{i 2}-d p^{2 i}\right) A^{1}$, and $d \zeta$ provides

$$
\begin{aligned}
& X_{i}\left(D_{n} G_{m}^{i j k} d t^{n} d p_{j k} d t^{m}+\frac{\partial H_{m n}^{i}}{\partial p_{j k}} d p_{j k} d t^{m} d t^{n}\right)+d p_{i j} d t^{j} K_{n}^{i m} d t^{n} X_{m} \\
& =X_{i}\left(D_{n} G_{m}^{i j k}+\frac{\partial H_{m n}^{i}}{\partial p_{j k}}+K_{n}^{j i} \delta_{m}^{k}\right) d p_{j k} d t^{m} d t^{n} .
\end{aligned}
$$

We have to take the $d^{34}$ term. The resulting equation is

$$
\begin{aligned}
\left(d p^{i 2}\right. & \left.-d p^{2 i}\right) A^{1}-\left(d p^{i 1}-d p^{1 i}\right) A^{2} \\
& =\left(D_{3} G_{4}^{i j k}-D_{4} G_{3}^{i j k}+2 \frac{\partial H_{43}^{i}}{\partial p_{j k}}+K_{3}^{j i} \delta_{4}^{k}-K_{4}^{i j} \delta_{3}^{k}\right) d p_{j k} \\
& =\left(D_{3} \gamma^{i j} \delta_{4}^{k}-D_{4} \gamma^{i j} \delta_{3}^{k}-2 \frac{\partial H_{34}^{i}}{\partial p_{j k}}+K_{3}^{j i} \delta_{4}^{k}-K_{4}^{i j} \delta_{3}^{k}\right) d p_{j k},
\end{aligned}
$$

using (7.4).

First let us take $i=1$ and select the coefficient of $d p^{21}$ which is a constant times $d p_{21}$. We obtain

$$
-A^{1}=-2 \frac{\partial H_{34}^{1}}{\partial p^{21}}=\frac{\partial^{2} J^{2}}{\partial p^{21} \partial p_{11}}
$$

using (7.6). Recall $J^{2}=N^{2}+W^{2}$.

Now, because $N$ satisfies (5.1),

$$
\frac{\partial}{\partial p^{21}} \frac{\partial N^{2}}{\partial p_{11}}=-\frac{\partial}{\partial p^{21}} \frac{2 N^{1}}{\partial p_{12}}=-\frac{\partial}{\partial p_{12}} \frac{\partial N^{1}}{\partial p^{21}}=-\frac{\partial}{\partial p^{12}} \frac{\partial N^{1}}{\partial p_{21}}=0 .
$$


From (2.34) we have

$$
\frac{\partial^{2} W^{2}}{\partial p^{21} \partial p_{11}}=-\frac{\partial S}{\partial u_{1}}
$$

Thus $A^{1}=\partial S / \partial u_{1}$.

Now we go back to (7.7) and take $i=3$, and look for the coefficients of $d p^{32}$. We obtain

$$
A^{1}=-2 \frac{\partial H_{34}^{3}}{\partial p^{32}}=-\frac{\partial^{2} J^{2}}{\partial p^{32} \partial p_{31}}
$$

By (5.1),

From (2.34), we get

$$
\frac{\partial N^{2}}{\partial p_{32}}=0
$$

$$
\frac{\partial W^{2}}{\partial p_{32} \partial p_{31}}=0 \text {. }
$$

Thus $A^{1}=0$ and so $\partial S / \partial u^{1}=0$ as (2.44) asserts.

We begin the proof of (2.45). Presumably (7.3) holds, but $J^{i}$ consists only of $N^{i}$, in other words, $S=0$. We have just learned that $A^{i}=0$. Let us examine the $d p_{21} d^{234}$ terms of (7.3). There $d \varepsilon$ has $\partial N^{1} / \partial p_{21}$ which is 0 because $N$ satisfies (5.1), and $\left.U\right\rfloor d \alpha$ has $-B_{2}$. What $d \zeta$ has must come from $d p_{21} d t^{1} H_{j k}^{i} d^{j k}$, so it provides nothing. So $B_{2}=0$, and all $B_{i}=0$.

Now we examine the $X_{3} d^{234}$ terms of (7.3). There $d \varepsilon$ provides $\partial N^{1} / \partial u^{3}$, and $\left.U\right\rfloor d \alpha$ has $C^{31}-C^{13}$. Those which $d \zeta$ provides must come from $X_{3} d E^{3}$. The only ttt terms in $d E^{3}$ lie in $\left(D_{m} H_{j k}^{3}\right) d t^{m} d^{j k}$, and so $d \zeta$ provides

$$
2 D_{2} H_{34}^{3}+2 D_{3} H_{42}^{3}+2 D_{4} H_{23}^{3} \text {. }
$$

Hence $\partial N^{1} / \partial u^{3}+C^{31}-C^{13}+(7.8)$ is 0 .

In the same way as we proved (7.6) we can show

$$
2 H_{23}^{3}=-\frac{\partial J^{1}}{\partial p_{34}} \text { and } 2 H_{42}^{3}=-\frac{\partial J^{1}}{\partial p_{33}} \text {. }
$$

Using these, and (7.6), we obtain

$$
\begin{aligned}
C^{31}-C^{13} & =-\frac{\partial N^{1}}{\partial u_{3}}-\left(-D_{4} \frac{\partial N^{1}}{\partial p_{34}}+D_{2} \frac{\partial N^{2}}{\partial p_{31}}-D_{3} \frac{\partial N^{1}}{\partial p_{33}}\right) \\
& =-\frac{\partial N^{1}}{\partial u_{3}}-D_{4} \frac{\partial N^{4}}{\partial p_{31}}-D_{2} \frac{\partial N^{2}}{\partial p_{31}}-D_{3} \frac{\partial N^{3}}{\partial p_{31}}
\end{aligned}
$$

We know from (3.7) that $D_{i} N^{i}=0$. Taking $\partial / \partial p_{31}$ of this equation, we get a new equation that says $C^{31}-C^{13}=0$. Thus $C^{i j}=C^{j i}$, in general.

That certainly implies that $U\rfloor d \alpha=0$. It also makes $U\rfloor \alpha=0$ (see(2.5)). Thus it makes $\left.\left.\ell_{U} \alpha=d(U\rfloor \alpha\right)+U\right\rfloor d \alpha\left[3\right.$, p. 172] Hence $\ell_{U} \alpha=d(d \theta)$, where $\theta$ is some 2-form, so $N(U)=0-d \theta$, and $N(U)$ is equivalent to the 0 -form, as we were to prove for (2.45). 
7.9. Corollary. Let $U$ be a vector field in $J^{1}(M, Q)$ such that $\ell_{U} \alpha=0$. Then $U=$ $U_{1}+U_{2}+U_{3}$, where $U_{1}$ is as in (7.21), $U_{2}$ is as in (7.22) and

where $C^{i j}=C^{j i}$.

$$
U_{3}=C^{i j} \frac{\partial}{\partial p_{i j}}
$$

Proof. Let $\varepsilon=U\rfloor \alpha$. Then $\varepsilon$ is Noetherian, and afortiori, dynamic. It is perhaps not a current. It might have $d u_{i}$ terms. If so, we can replace $d u_{i}$ by $X_{i}+p_{i j} d t^{j}(2.7)$, obtaining an $\varepsilon_{1}=\varepsilon+\zeta$, where $\zeta$ vanishes on all motions, and $\varepsilon_{1}$ is a current. We apply (2.3), and deduce that

$$
\varepsilon=\varepsilon_{\Phi}+\varepsilon_{\Psi}+\varepsilon_{I}+\varepsilon_{W}+\varepsilon_{N}+\zeta_{1}+\eta,
$$

where $\zeta_{1}=0$ on all motions, and $\eta$ is exact. By (2.44), we know $\varepsilon_{W}$ is Noetherian and may be combined with $\varepsilon_{\Psi}$, and $\left.\varepsilon_{\Psi}=U_{2}\right\rfloor \alpha-d \psi$. Here $\varepsilon_{\Phi}$ is of the form $\left.U_{1}\right\rfloor \alpha$, and $\varepsilon_{I}$ is exact. Thus

$$
\left.\left(U-U_{1}-U_{2}\right)\right\rfloor \alpha=\varepsilon_{N}+\zeta_{1}+\eta_{1},
$$

where $\eta_{1}$ is again exact. This is the situation at the start of the proof of (2.45). We then deduced that $U-U_{1}-U_{2}=C^{i j} \partial / \partial p_{i j}$ with $C^{i j}=C^{j i}$.

Remark. Calculation of $\ell_{U} \alpha$ with $U$ as above shows that the $f_{i}$ involved in $U_{2}$ satisfy $\partial f_{i} / \partial t^{j}=\partial f_{j} / \partial t^{i}$, so that $U_{2}$ is a gauge transformation.

\section{The Energy-Momentum Tensor is Dynamic but not Noetherian}

Let $A^{j}=g^{j m}$ in (2.31). Then for each $m$, the

$$
\Phi^{i m}=p_{k j} g^{j m}\left(p^{i k}-p^{k i}\right)+\frac{1}{2} g^{i m} p_{j k}\left(p^{j k}-p^{k j}\right)
$$

are the components of a Noetherian form, based on the translation $\partial / \partial t_{m}$ in space-time.

Now take $S=g^{m i} u_{i}$. Then (from (2.34))

$$
W^{i m}=g^{m k} p_{k j}\left(p^{j i}-p_{i j}\right)=-g^{j m} p_{j k}\left(p^{i k}-p^{k i}\right)
$$

are the components of a dynamic current which we now know is not equivalent to any Noetherian form. The sum

$$
T^{i m}=\frac{1}{2} g^{i m} p_{j k}\left(p^{j k}-p^{k j}\right)-g_{k n}\left(p^{i k}-p^{k i}\right)\left(p^{m n}-p^{n m}\right)
$$

gives the components of the energy-momentum tensor (cf. [4]) for the case of an electromagnetic field. Each row (and each column) of $T$ is a dynamic form which is not equivalent to a Noetherian form.

The dynamic currents of type (2.34) resemble Noetherian forms in that they have the form (2.6): $\left.\varepsilon_{W}=U\right\rfloor \alpha$, where $U=D_{i} S \partial / \partial u_{i}$.

\section{References}

1 Arens, Richard: The dynamic differential forms of the Klein-Gordon field and the conformal group. (to appear) 
2. Bessel-Hagen, E: Uber die Erhaltungssätze der Elektrodynamik. Math. Ann. 84, 258-275 (1921)

3. Bishop R. L. Goldberg, S. I.: Tensor analysis on manifolds. New York: Macmillan 1968

4. Einstein, A., Rosen, N.: The particle problem in the general theory of relativity. Phys. Rev. 48, 73-77 (1935)

5. Goldschmidt, H., Sternberg, S.: The Hamilton-Cartan formalism in the calculus of variations. Ann. Inst. Fourier, Grenoble 23, 203-267 (1973)

6. Golubitzsky M., Guillemin, V.: Stable mappings and their singularities. C. T. M. Vol. 14, Berlin, Heidelberg New York: Springer 1973

7. Wentzel, G.: Quantum theory of fields, New York: Interscience Publishers 1949

Communicated by R. Haag

Received February 5, 1983; in revised form April 11, 1983 